\title{
Genotype/phenotype correlation of the G85E mutation in a large cohort of cystic fibrosis patients
}

\author{
K. Decaestecker*, E. Decaestecker", C. Castellani", M. Jaspers ${ }^{+}$, H. Cuppens ${ }^{+}$, K. De Boeck*
}

Genotypelphenotype correlation of the G85E mutation in a large cohort of cystic fibrosis patients. K. Decaestecker, E. Decaestecker, C. Castellani, M. Jaspers, H. Cuppens, K. De Boeck. C) ERS Journals Ltd 2004.

ABSTRACT: In this European study, the phenotype in 68 patients, homozygous or compound heterozygous for the G85E mutation, was investigated.

Each index case was compared with two cystic fibrosis (CF) patients from the same clinic, matched for age and sex: one with pancreatic sufficiency (PS) and one with pancreatic insufficiency (PI).

When comparing 31 G85E/F508del and F508del/F508del patients, there were no differences in median age at diagnosis, mean sweat chloride value, most recent weight for height, most recent forced expiratory volume in one second \% predicted, prevalence of chronic Pseudomonas aeruginosa colonisation and typical CF complications. However, PI was less frequent in the G85E/F508del group. Comparison of 55 G85E patients (with second mutation known and not classified as mild) with PS controls $(n=44)$ showed that the G85E patients had a significantly higher sweat chloride, more often failure to thrive at diagnosis, higher prevalence of PI, worse current weight for height, higher prevalence of chronic $P$. aeruginosa colonisation and liver cirrhosis. Pulse-chase experiments revealed that G85E cystic fibrosis transmembrane conductance regulator failed to mature on a $\mathrm{M470}$ as well as on a V470 background. Therefore, G85E is a class II mutation.

Although there is variability in its clinical presentation, G85E mutation results in a severe phenotype.

Eur Respir J 2004; 23: 679-684.
*Dept of Pediatrics, ${ }^{\#}$ Laboratory of Aquatic Ecology, and ${ }^{+}$Dept for Human Genetics, University of Leuven, Leuven, Belgium. ${ }^{+}$Ospedale Civile Maggiore, Verona, Italy.

Correspondence: K. De Boeck

University of Leuven

Cystic Fibrosis Center of Leuven

Herestraat 49

B-3000 Leuven

Belgium

Fax: 3216343842

E-mail: christiane.deboeck@uz.kuleuven.ac.be

Keywords: Cystic fibrosis

cystic fibrosis transmembrane conductance

regulator

G85E mutation

genotype-phenotype

mutation

Received: February 102003

Accepted after revision: January 222004
Cystic fibrosis $(\mathrm{CF})$ is the most common lethal, congenital disease in White populations. It is caused by mutations on both genes coding for the cystic fibrosis transmembrane conductance regulator (CFTR) protein. The gene spans $\sim 250 \mathrm{~kb}$ on the long arm of chromosome 7 and encodes a chloride channel regulated by cyclic adenosine monophosphate. Defects in the CFTR protein are responsible for abnormal chloride transport across the apical membrane of exocrine epithelial cells and result in a highly variable clinical expression from mild to very severe disease [1, 2]. Severe phenotypes are usually associated with high concentrations of sweat chloride, early onset of pancreatic insufficiency (PI) and severe lung disease. Mild phenotypes are associated with lower sweat chloride concentrations, pancreatic sufficiency (PS), variable lung disease and no history of meconium ileus. In addition, patients with atypical disease and normal sweat chloride values, as well as patients with disease manifestations limited to one organ, have been described. Whether these individuals can be considered affected by CF or not has not yet been fully elucidated [2]. Within the group of patients with classical $\mathrm{CF}$ and sweat chlorides $>60 \mathrm{mEq} \cdot \mathrm{L}^{-1}$, the pancreatic status of the patient can be taken as a marker of disease severity: patients with PS have significantly lower sweat chlorides [2], slower progression of lung disease [3,4] and usually carry at least one mild mutation [5].

To date, $>1,000$ different mutations in the CFTR gene have already been reported by the Cystic Fibrosis Genetic Analysis
Consortium [6]. The genotype-phenotype correlation is not known for many rare mutations. Worldwide, of all mutations reported, the G85E mutation has a frequency $\sim 0.2 \%$. It is more prevalent in the Mediterranean region (Spain 1\%, Italy $1.7 \%)[7,8]$. The G85E mutation is a missense mutation: in exon 3, at nucleotide position 386, guanine is replaced by adenosine, resulting in the substitution of glycine by negatively charged glutamic acid in the first membrane-spanning domain [9]. Earlier reports about G85E concern a small number of patients. It is not clear whether the mutation correlates with a mild, a severe or a variable phenotype [8-11].

The purpose of the present study was to determine the clinical outcome of $\mathrm{CF}$ patients, homozygous or compound heterozygous for the G85E mutation, in a large study group. In addition, the effect of G85E mutation constructs on CFTR protein expression was evaluated in in vitro cell lines.

\section{Materials and methods}

The physicians cooperating in the $\mathrm{CF}$ thematic network [12] were contacted by e-mail. They were asked to report clinical data on their patients homozygous or compound heterozygous for the G85E mutation and, per index case, on two control CF patients. The control patients needed to have a firm diagnosis of $\mathrm{CF}$, including a sweat chloride $>60 \mathrm{mEq} \cdot \mathrm{L}^{-1}$. 
They were matched for sex and age, their birth date being as close as possible to the index case. One control patient needed to have PI and one control patient needed to have PS. Exocrine pancreatic insufficiency was defined as $>7 \mathrm{~g}$ of faecal fat loss per day and/or fat absorption $<93 \%$ on 3 -day faecal fat balance.

To describe the severity of the disease, the following clinical variables were used: age at diagnosis; sweat chloride concentration using pilocarpine iontophoresis [13]; presenting symptom (meconium ileus, failure to thrive, steatorrhea, respiratory disease); most recent weight for height; most recent forced expiratory volume in one second (FEV1) \% predicted; presence of chronic Pseudomonas aeruginosa colonisation; and occurrence of complications such as liver cirrhosis, cystic fibrosis-related diabetes (CF-RD), distal intestinal obstruction syndrome (DIOS) and pancreatitis.

Weight-for-height percentiles were calculated using the standards of PRADER et al. [14]. Chronic P. aeruginosa colonisation was defined as stated in the European consensus report: presence of $P$. aeruginosa in the bronchial tree for 6 months, based on at least three positive sputum cultures at 1-month intervals [15]. Liver cirrhosis was reported when a firm (left) liver (lobe) was felt and ultrasound confirmed signs of cirrhosis or when there was a large palpable spleen. CF-RD refers to disturbed glucose metabolism treated with insulin. DIOS was defined as intermittent abdominal pain and a palpable faecal mass in the right abdominal quadrant, leading to hospital admission for medical or surgical treatment. Pancreatitis was considered as any acute episode of abdominal pain associated with serum amylase levels $\geqslant 1.5$ times above the upper limit of normal for the individual laboratories.

For evaluation of lung function, FEV1 values were used at their most recent assessment and they were determined as recommended by the ATS consensus [16]. All values were expressed as percent predicted value by QUANJER and PORSBOOM [17].

In order to have a clear-cut evaluation of the influence of the G85E mutation, a primary analysis for G85E/F508del patients versus F508del/F508del PI patients was performed. The G85E patients were also compared with the groups with PI and PS. Mild mutations confer the PS phenotype in a dominant way [5]. From the group of G85E patients, thirteen with unknown second mutation or with a second mutation known to be associated with PS were eliminated.

For continuous, normally distributed data, results are expressed as mean \pm SEM and compared by paired t-test. Skewed data are presented as median (interquartile range). For continuous variables, the Wilcoxon signed-ranks test was used. For analysis of binary variables, Fisher's exact test was used.

Apart from studying the phenotype in patients carrying the G85E mutation, the G85E mutation was expressed in cell lines, and the influence of the mutation on CFTR protein biosynthesis and expression in vitro was studied. This technique helps to determine which mutation class G85E belongs to, at least in the chosen expression system [18]. G85E-CFTR cDNA/pcDNA3 expression vectors, either on a M470-CFTR or V470-CFTR background, were made by means of the Transformer ${ }^{\mathrm{TM}}$ Site-Directed Mutagenesis Kit (Clontech, Palo Alto, CA, USA), using the mutagenesis primer 5'-GGA GAT TTA TGT TCT ATG AAA TCT TTT-3', according to previously described protocols [19]. The complete CFTRcoding region of these constructs was verified by sequencing. Expression vectors were transiently transfected in COS-1 cells. After metabolic labelling of transfected cells, pulse-chase experiments were performed over different time periods according to previously described protocols [19], except for immunoprecipitation of CFTR with a C-terminal CFTR antibody (R\&D systems, Minneapolis, MN, USA).

\section{Results}

Twenty-one centres in seven European countries contributed data. Data were collected on the phenotype of 68 patients homozygous or compound heterozygous for the G85E mutation. Some centres were unable to supply data on matched PS patients.

\section{Genotypes}

The genotypes of all patients are presented in table 1 .

Out of 68 patients in the G85E group, 34 carried the G85E/ F508del mutations. In 26 patients, the second mutation was known but was not the F508del: in 21 of these patients the second mutation was known to be associated with severe disease; and in two of these patients the second mutation was considered as a mild mutation. In eight patients the second mutation was not known. In three patients the phenotype of the second mutation was not known. One index case was homozygous for the G85E mutation and was PS. All but two patients in the G85E group were Caucasian: one was Caucasian/African (Antilles) and one was African.

All patients in the PI group carried at least one F508del mutation. Fifty-eight of the 68 PI patients were F508del homozygous. In eight the second mutation was known.

In the PS group only one patient was F508del homozygous. Thirty-three patients were F508del heterozygous, but in 15 of them the second mutation was not known. Twelve patients had known but non-F508del mutations on both CFTR genes. In nine patients neither mutation was known. At least 13 patients carried a mutation known to confer mild disease $[18,20]$.

\section{G85E/F508del compared to F508dellF508del}

This analysis concerns 31 patients and is presented in table 2: three of the 34 patients with G85E/F508del genotype in whom the PI control patient did not have a F508del/ F508del genotype were excluded from analysis. Mean current age, median age at diagnosis, mean sweat chloride value, most recent weight for height, most recent FEV1 \% pred, per cent of patients with chronic $P$. aeruginosa colonisation and typical CF complications are reported in table 2, and did not differ between the two subgroups. However, PI was less frequent in the G85E/F508del group. In addition, at time of diagnosis, steatorrhea and failure to thrive were less frequent in $\mathrm{G} 85 \mathrm{E} /$ F508del patients. Chronic or recurrent respiratory symptoms occurred equally frequently in both groups.

\section{G85E patients (with second mutation known and not classified as mild) compared to PI and PS matched controls}

Comparison of this larger G85E patient group with their PI controls $(n=55$; table 3$)$ showed the same results as the comparison between the G85E/F508del subgroup versus the F508del/ F508del group. No differences in mean current age, median age at diagnosis, median sweat chloride value, current weight for height, most recent FEV1 \% pred, prevalence of $P$. aeruginosa colonisation nor CF complications were found. Again, there was a lower percentage of patients having PI and steatorrhea or failure to thrive at diagnosis.

Comparison of the G85E group with PS controls ( $n=44$; table 3 ) showed that the G85E patients have a significantly higher sweat chloride $(\mathrm{p}<0.0018)$, more often present 
Table 1.-Cystic fibrosis transmembrane conductance regulator genotypes of patients in the three study groups

\begin{tabular}{|c|c|c|c|c|c|}
\hline \multicolumn{2}{|c|}{ G85E } & \multicolumn{2}{|l|}{ PI } & \multicolumn{2}{|l|}{ PS } \\
\hline Genotype & Subjects $n$ & Genotype & Subjects $n$ & Genotype & Subjects $n$ \\
\hline G85E/F508del ${ }^{\#}$ & 34 & 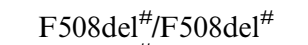 & 58 & F508del ${ }^{\# / \text { unknown }}$ & 15 \\
\hline G85E/unknown & 8 & F508del ${ }^{\# / \text { unknown }}$ & 2 & $\mathrm{~F} 508 \mathrm{del}^{\#} / 3849+10 \mathrm{kbC} \rightarrow \mathrm{T}^{\oplus}$ & 5 \\
\hline G85E/G542X & 5 & $\mathrm{~F} 508 \mathrm{del}^{\#} / 1717-1 \mathrm{G} \rightarrow \mathrm{A}$ & 1 & $\mathrm{~F} 508 \mathrm{del}^{\# / R} 117 \mathrm{H}^{\oplus}$ & 3 \\
\hline G85E/W1282X \# & 4 & F508del $/ \mathrm{N} 1303 \mathrm{~K}^{\#}$ & 1 & T338I/L1065P & 2 \\
\hline G85E/I507del ${ }^{\#}$ & 3 & F508del*/H139R & 1 & $\mathrm{E} 585 \mathrm{X} / 3272-26 \mathrm{~A} \rightarrow \mathrm{G}^{\bullet}$ & 2 \\
\hline G85E/R1162X & 3 & F508del $/$ R1066C & 1 & $2183 \mathrm{AA} \rightarrow \mathrm{G} / 2789+5 \mathrm{G} \rightarrow \mathrm{A}$ & 2 \\
\hline $\mathrm{G} 85 \mathrm{E} / 2183 \mathrm{AA} \rightarrow \mathrm{G}$ & 2 & F508del ${ }^{\#} / \mathrm{G} 542 \mathrm{X}^{\#}$ & 1 & $\mathrm{~F} 508 \mathrm{del}^{\#} / 711+5 \mathrm{G} \rightarrow \mathrm{A}$ & 1 \\
\hline G85E/G85E & 1 & $\mathrm{~F} 508 \mathrm{del}^{\#} / 712-1 \mathrm{G} \rightarrow \mathrm{T}$ & 1 & $\mathrm{~F} 508 \mathrm{del}^{\#} / \mathrm{D} 1152 \mathrm{H}^{\oplus}$ & 1 \\
\hline G85E/E585X \# & 1 & $\mathrm{~F} 508 \mathrm{del}^{\#} / 621+1 \mathrm{G} \rightarrow \mathrm{T}$ & 1 & $\mathrm{~F} 508 \mathrm{del}^{\#} / 1898+3 \mathrm{~A} \rightarrow \mathrm{G}$ & 1 \\
\hline $\mathrm{G} 85 \mathrm{E} / 711+1 \mathrm{G} \rightarrow \mathrm{T}^{\#}$ & 1 & F508del ${ }^{\#} / 1898+1$ & 1 & $\mathrm{~F} 508 \mathrm{del}^{\#} / \mathrm{R} 347 \mathrm{H}^{\bullet}$ & 1 \\
\hline $\mathrm{G} 85 \mathrm{E} / 712-1 \mathrm{G} \rightarrow \mathrm{T}^{\#}$ & 1 & Total & 68 & $\mathrm{~F} 508 \mathrm{del}^{\#} / 2789+5 \mathrm{G} \rightarrow \mathrm{A}$ & 1 \\
\hline $\mathrm{G} 85 \mathrm{E} / 621+1 \mathrm{G} \rightarrow \mathrm{T}^{\#}$ & 1 & & & $2789+5 \mathrm{G} \rightarrow \mathrm{A} / ?$ & 1 \\
\hline G85E/W496X & 1 & & & F508del ${ }^{\#} / \mathrm{N} 1303 \mathrm{~K}^{\#}$ & 1 \\
\hline G85E/N1303K $\#$ & 1 & & & T388I/R1158X & 1 \\
\hline $\mathrm{G} 85 \mathrm{E} / 711+5 \mathrm{G} \rightarrow \mathrm{A}^{\bullet}$ & 1 & & & $3272-26 \mathrm{~A}>\mathrm{G}^{\top} / \mathrm{E} 822 \mathrm{X}$ & 1 \\
\hline G85E/R334W & 1 & & & F508del ${ }^{\#} / \mathrm{R} 334 W^{\oplus}$ & 1 \\
\hline Total & 68 & & & $574 \mathrm{del} \mathrm{A} / 2789+5 \mathrm{G} \rightarrow \mathrm{A}$ & 1 \\
\hline & & & & $\mathrm{F} 508 \mathrm{del}^{\#} / 3272-26 \mathrm{~A} \rightarrow \mathrm{G}^{\bullet}$ & 1 \\
\hline & & & & $\mathrm{F} 508 \mathrm{del}^{\#} / \mathrm{R} 352 \mathrm{Q}$ & 1 \\
\hline & & & & $\mathrm{F} 508 \mathrm{del}^{\#} / 3272-26 \mathrm{~A}>\mathrm{G}^{\bullet}$ & 1 \\
\hline & & & & R334W $/ 444$ delA & 1 \\
\hline & & & & $\mathrm{L} 206 \mathrm{~W} / 3272-26 \mathrm{~A} \rightarrow \mathrm{G}^{\oplus}$ & 1 \\
\hline & & & & F508del ${ }^{\#} / \mathrm{F} 508 \mathrm{del}^{\#}$ & 1 \\
\hline & & & & L206W/? & 1 \\
\hline & & & & Unknown/unknown & 9 \\
\hline & & & & Total 56 & \\
\hline
\end{tabular}

PI: pancreatic insufficiency; PS: pancreatic sufficiency. " : mutation associated with severe disease; ": mutation associated with "mild" disease. Classifying mutations as severe and mild may be controversial because for many mutations functional studies have not been performed. The mutations have been marked in accordance with other authors' findings $[1,2,6,18,20,21,24,26]$.

symptoms of steatorrhea and failure to thrive at diagnosis $(\mathrm{p}<0.0001)$, higher prevalence of pancreatic insufficiency $(\mathrm{p}<0.0001)$, worse current weight for height $(\mathrm{p}<0.02)$, higher prevalence of chronic $P$. aeruginosa colonisation $(\mathrm{p}<0.0083)$ and liver cirrhosis $(\mathrm{p}<0.05)$. The most recent FEV1 \% pred was $69 \pm 4.8 \%$ in the G85E group and $85 \pm 6.6 \%$ in the PS group $(\mathrm{p}=0.08)$. Median age at diagnosis was 22 months in the
G85E group and 44 months in the PS group ( $\mathrm{p}=0.13)$. CF-RD was more frequent (11 versus $2 \%, \mathrm{p}=0.1$ ).

If all G85E patients were considered $(n=68)$ in the analysis, these last differences again reached statistical significance (data not shown). Three patients of the G85E group died: two patients died at the age of $10 \mathrm{yrs}$, of whom one after Burkholderia cepacia colonisation; and one patient died at

Table 2. - Current age and clinical variables in a group of European cystic fibrosis (CF) patients heterozygous for G85E and F508del compared to CF patients homozygous for F508del

G85E/F508de

Subjects $n$

Current age yrs

Age at time of diagnosis months

Sweat chloride $\mathrm{mEq} \cdot \mathrm{L}^{-1}$

Presenting symptoms

Steatorrhea

Failure to thrive

Meconium ileus

Respiratory

Pancreatic insufficiency

Most recent weight for height percentile

Most recent FEV1 \% pred

Chronic $P$. aeruginosa colonisation

Complications

Liver cirrhosis

CF-DM

DIOS

Pancreatitis $n$

F508del/F508del

G85E/F508del versus F508del/F508del

$19.5 \pm 7.2(2-40)$

$5(2-40)$

$100(90-110)$

$\begin{array}{cc}38 & 86 \\ 38 & 70 \\ 4 & 7 \\ 82 & 62 \\ 60 & 100 \\ 60 \pm 5 & 58 \pm 6 \\ 69 \pm 5 & 65 \pm 6 \\ 41 & 45\end{array}$
$0.05^{\#}$
$0.2^{\circ}$
$0.95^{\circ}$
$0.0003^{+}$
$0.017^{+}$
$0.38^{+}$
$0.88^{+}$
$<0.001^{+}$
$0.68^{\#}$
$0.78^{\#}$
$0.33^{+}$
$0.119^{+}$
$0.298^{+}$
$0.16^{+}$
$0.5^{+}$

Data are presented as mean \pm SD (range), median (interquartile range), mean \pm SEM or $\%$ unless otherwise stated. Subjects were matched for centre, sex and age. FEV1: forced expiratory volume in one second; pred: predicted; P. aeruginosa: Pseudomonas aeruginosa; CF-RD: cystic fibrosis-related

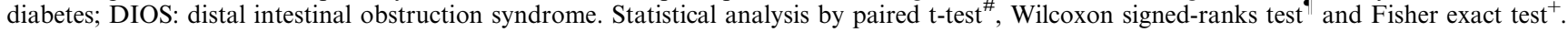


Table 3. - Current age and clinical variables in a group of European cystic fibrosis (CF) patients, carrying the G85E mutation and a known second mutation not associated with pancreatic sufficiency (PS), compared to two groups of CF patients not carrying the G85E mutation and having either pancreatic insufficiency (PI) or PS

\begin{tabular}{|c|c|c|c|c|}
\hline & G85E & PS & PI & G85E versus $\mathrm{PS}$ \\
\hline Subjects $n$ & 55 & 44 & 55 & \\
\hline Current age yrs & $18.2 \pm 9(2-51)$ & $18.2 \pm 9(2-52)$ & $18.9 \pm 9(3-46)$ & $0.06^{\#}$ \\
\hline Age at time of diagnosis months & $22(3-124)$ & $44(6-169)$ & $6(2-28)$ & $0.13^{\top}$ \\
\hline Sweat chloride $\mathrm{mEq} \cdot \mathrm{L}^{-1}$ & $106(95-115)$ & $100(78-109)$ & $102(94-110)$ & $0.0018^{\top}$ \\
\hline \multicolumn{5}{|l|}{ Presenting symptoms } \\
\hline Steatorrhea & 40 & 5 & 84 & $0.0001^{+}$ \\
\hline Failure to thrive & 40 & 14 & 71 & $0.0001^{+}$ \\
\hline Meconium ileus & 2 & 0 & 12 & $0.49^{+}$ \\
\hline Respiratory & 78 & 74 & 63 & $0.17^{+}$ \\
\hline Pancreatic insufficiency & 54 & 0 & 100 & $<0.0001^{+}$ \\
\hline Most recent weight for height percentile & $57 \pm 4$ & $72 \pm 5$ & $58 \pm 5$ & $0.02^{\#}$ \\
\hline Most recent FEV1 \% pred & $69 \pm 5$ & $85 \pm 7$ & $66 \pm 5$ & $0.08^{\#}$ \\
\hline Chronic $P$. aeruginosa colonisation & 42 & 23 & 46 & $0.0083^{+}$ \\
\hline \multicolumn{5}{|l|}{ Complications } \\
\hline Liver cirrhosis & 9 & 0 & 11 & $<0.05^{+}$ \\
\hline CF-RD & 11 & 2 & 13 & $0.1^{+}$ \\
\hline DIOS & 15 & 7 & 9 & $0.11^{+}$ \\
\hline Pancreatitis $n$ & 2 & 2 & 0 & $0.55^{+}$ \\
\hline
\end{tabular}

Data are presented as mean \pm SD (range), median (interquartile range), mean \pm SEM or $\%$ unless otherwise stated. Subjects were matched for centre, sex and age. FEV1: forced expiratory volume in one second; pred: predicted; P. aeruginosa: Pseudomonas aeruginosa; CF-RD: cystic fibrosis-related diabetes; DIOS: distal intestinal obstruction syndrome. Statistical analysis by paired t-test ${ }^{\#}$, Wilcoxon signed-ranks test ${ }^{\top}$ and Fisher exact test $^{+}$.

the age of $26 \mathrm{yrs}$ after lung transplantation. This further documents the severe lung disease in the G85E patients. The frequency of pancreatic insufficiency was $57 \%$ in the total G85E group. Within the total G85E group, the age of the patients with PI was $23.0 \pm 1.7$ yrs and of the patients with PS $16.1 \pm 1.7$ yrs. PI patients were significantly older than PS patients $(\mathrm{p}=0.0045)$.

\section{Sibling pairs}

There were six sibling pairs in the G85E group. Two sibpairs, all compound heterozygous for the G85E and F508del mutations, were discordant for pancreatic disease manifestation. Out of 23-yr-old dizygotic twins, the boy suffered from PI, had a sweat chloride level of $106 \mathrm{mEq} \cdot \mathrm{L}^{-1}$, a weight for height percentile of 55 and liver cirrhosis. His twin sister was $\mathrm{PS}$, had a sweat chloride value of $84 \mathrm{mEq} \cdot \mathrm{L}^{-1}$ and a weight for height percentile of 100 . Both were chronically colonised with $P$. aeruginosa. The other sib-pair discordant for pancreatic function were 21 and 23 yrs old. The younger PI sib had a sweat chloride value of $97 \mathrm{mEq} \cdot \mathrm{L}^{-1}$ and a weight for height percentile of 90 . The PS sib had a sweat chloride value of $116 \mathrm{mEq} \cdot \mathrm{L}^{-1}$ and a weight for height percentile of 30 . Neither were chronically colonised with $P$. aeruginosa.

\section{In vitro functional properties of G85E-CFTR}

The degree of G85E-CFTR maturation was investigated. It is known that polymorphic loci, such as M470V, can affect the properties of CFTR [19]. However, the haplotype background of G85E-CFTR genes is mostly unknown. G85E was, therefore, studied either on a M470 or V470 background. G85E-V470-, G85E-M470- and wildtype-V470-CFTR were transiently expressed in COS cells. Pulse-chase experiments, lasting $30 \mathrm{~min}, 1 \mathrm{~h} 30 \mathrm{~min}$ and $3 \mathrm{~h} 30 \mathrm{~min}$, showed maturation to the complex-glycosylated C-form for wild type-V470CFTR. In contrast, G85E-V470- and G85E-M470-CFTR

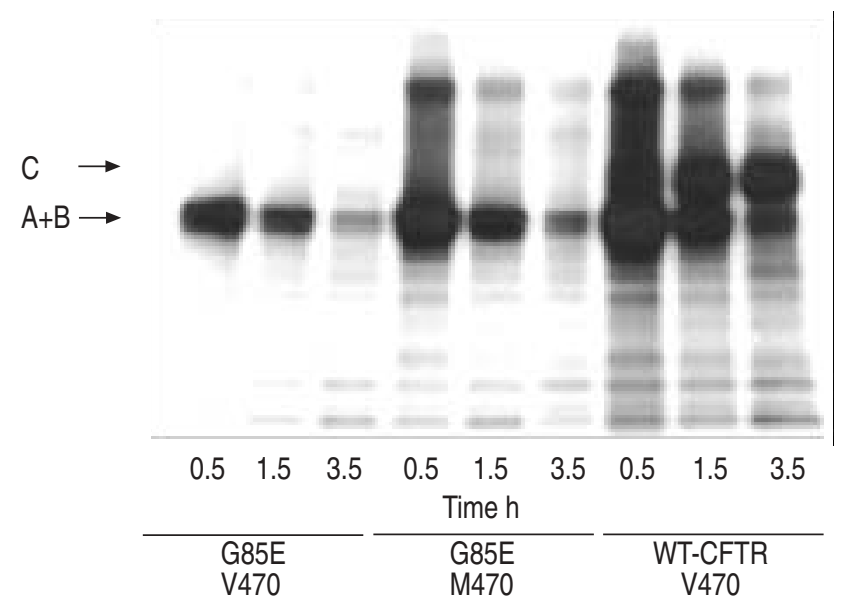

Fig. 1.-Analysis of biogenesis and degradation of G85E-cystic fibrosis transmembrane conductance regulator (CFTR). COS cells transfected with either G85E-V470-, G85E-M470- or wild type-V470CFTR were metabolically labelled and chased for the times indicated. Subsequently, the cells were lysed, CFTR was immunoprecipitated with a monoclonal antibody directed to the $\mathrm{COOH}$ terminus of CFTR, and analysed by PAGE and autoradiography. The positions of the primary translated (A-form), core-glycosylated (B-form) and complex-glycosylated (C-form) CFTR products are indicated.

failed to mature to the complex-glycosylated C-form at all time periods (fig. 1).

\section{Discussion}

$\mathrm{CF}$ patients with the G85E mutation have a severe phenotype. When comparing G85E/F508del and F508del/ F508del patients, there are no differences in age at diagnosis, sweat chloride value, parameters evaluating lung disease, most recent weight for height, nor $\mathrm{CF}$ complications. Conversely, comparing G85E patients with PS patients it 
has been shown here that the G85E group has more severe disease when considering the same parameters. Conversely, there are some differences with classical PI patients: $43 \%$ of the G85E patients are PS; and steatorrhea and failure to thrive are less frequent presenting symptoms. Disease variability for pancreatic function is present.

Within classical $\mathrm{CF}$, the pancreatic status differentiates mild and severe CF disease [3-5, 21]. PS patients have a milder expression than PI patients, with regards to sweat chloride value, mean patient age and pulmonary evolution $[22,23]$. Moreover, classical PS patients seem to be intermediate between PI and non-classical CF patients with regards to sweat chloride values and nasal potential difference measurements [24]. The pancreatic phenotype is thought to be more dependent on the CFTR genotype than the pulmonary phenotype, for which the environment and modifying genes play a larger role $[2,5,18,20,25,26]$. In the total G85E group studied, the frequency of PI was $57 \%$. In patients carrying mutations like F508del, N1303K and W1282X, which correlate with a severe phenotype, $>95 \%$ have PI [21]. In patients carrying mutations such as $\mathrm{R} 117 \mathrm{H}$, A455E, R334W and $3849+10 \mathrm{~kb} \mathrm{C}>\mathrm{T}$, which are reported to correlate with a milder phenotype, $40-87 \%$ have PS [21, 27].

Familial concordance of pancreatic function within a family is high [26]. Indeed, only two of six sibling pairs in this study were discordant for pancreatic status. Intra-familial discordance for pancreatic status has, however, been reported previously [28]. Conversely, patients may be PS at diagnosis and evolve towards PI [22]. In accordance with this report, the G85E PS patients in the present study are significantly younger than the G85E PI patients.

Modifying genes can alter the clinical presentation [29]. Indeed, modifying genes for meconium ileus have been identified in region 19q13.2 [30]. Modifying genes can, at present, not yet be studied, but they could explain the rather infrequent occurrence of meconium ileus in the larger group of G85E patients.

In a previous study, it was found that G85E-CFTR fails to mature and, therefore, is a class II mutation [31]. In general, class II mutations result in severe CF disease [18]. The heterogeneity in disease severity in the group of G85E-CF patients is thus remarkable for a class II mutation, unless if it is conferred by the mutation on the other chromosome. It is known that polymorphic loci such as $\mathrm{T}_{\mathrm{n}}, \mathrm{TG}_{\mathrm{m}}, \mathrm{M} 470 \mathrm{~V}$, affect the properties of CFTR. However, the haplotype background of G85E-CFTR genes is mostly unknown. G85E-CFTR was, therefore, studied both on a M470 and a V470 background, in order to investigate if G85E-CFTR properties are affected by the amino acid found at the M470V position and in this way could explain the observed disease variability. Both G85EM470- and G85E-V470-CFTR, however, completely failed to mature. On the basis of the present in vitro findings, as well as on the basis of the clinical findings in the patient group, G85E can be classified as a mutation associated with severe disease.

A variable such as the age at diagnosis may be biased by the introduction of screening programmes that may artificially lower the age at diagnosis in PS patients. Even so, the age at diagnosis was lower in G85E patients, supporting the idea of a more severe phenotype. Respiratory disease is the most important cause of morbidity and mortality in $\mathrm{CF}$ [20]. In the present study G85E patients have more severe lung disease as assessed both by more frequent chronic $P$. aeruginosa infection and worse most recent FEV1\% pred.

In conclusion, G85E is associated with a severe phenotype. The much larger patient sample compared to previous reports makes a correct evaluation of the phenotype more likely. There is some variability in the phenotypic expression of pancreatic disease. The aetiology of this is uncertain but modifying genes and environmental factors could be responsible.
Acknowledgements. European G85E survey contributors: T. Bienvenu (Hôpital Cochin, Paris, France); R. Cabanas (Hospital Clinico, Santiago, Spain); T. Casals (Hospital Duran I Reynals, Barcelona, Spain); G. Castaldo (Universita Federico II - Ceinge, Napoli, Italy); C. Castellani (Ospedale Civile Maggiore, Verona, Italy); J. Dapena (Hospital U. "Virgen del Rocio", Sevilla, Spain); K. De Boeck (University Hospital Gasthuisberg, Leuven, Belgium); E. Dequeker (European CF Thematic Network, Dept for Human Genetics, Leuven. Belgium); G. Evans-Jones (The Countess of Chester Hospital NHS Trust, Chester, UK); D. Feldmann (Hôpital A. Trousseau, Paris, France); I. Galeva (Pediatric Clinic State University Hospital, Sofia, Bulgaria); A. Göçmen (Hacettepe Univ. Pediatric Chest Diseases, Ankara, Turkey); E. Gunn (Papworth Hospital, Cambridge, UK); J. Manzanares (Hospital Universitario "12 de Octubre", Madrid, Spain); C. Olveira (C.H. Carlos Haya, Malaga, Spain); R. Padoan (Istituti Clinici di Perfezionamento, Milano, Italy); G. Picherot (Hôpital St. Nazaire, France); L. Romano (Istituto Giannina Gaslini, Geneva, Italy); J.L. Seculi (Hospital S. Joan de Deu, Barcelona, Spain); J. Sirvent (C.H. Juan Canalejo, Coruna, Spain); G. Taccetti, Centro Regionale Toscano Fibrosi Cistica, Firenze, Italy); B. Togores (Hospital Son Dureta, Palma Spain); C. Vazquez (Hopital de Cruces, Baracaldo, Spain).

\section{References}

1. Mickle J, Cutting G. Genotype-phenotype relationships in cystic fibrosis. Med Clin N Am 2000; 84: 597-607.

2. Zielenski J. Genotype and phenotype in cystic fibrosis Respiration 2000; 67: 117-133.

3. Gaskin K, Gurwitz D, Durie PR, Levison H. Forstner G. Improved respiratory prognosis in patients with cystic fibrosis with normal fat absorption. J Pediatr 1982; 100: 857-862.

4. Corey M, Edwards L, Levison H, Knowles M. Longitudinal analysis of pulmonary function decline in patients with cystic fibrosis. J Pediatr 1997; 131: 809-814.

5. Kristidis P, Bozon D, Corey M, et al. Genetic determination of exocrine pancreatic function in cystic fibrosis. Am J Hum Genet 1992; 50: 1178-1184.

6. Cystic Fibrosis Mutation Database. www.genet.sickkids. on.ca/cftr. Date last updated: October 2 2003. Date last accessed: February 162004.

7. Friedman K, Blalock M, Heim R, Silverman L. Relatively high prevalence of the CFTR mutations G85E and 1154insTC. Hum Mut 1995; 6: 95-96.

8. Vazquez C, Antinolo G, Casals T, et al. Thirteen cystic fibrosis patients, 12 compound heterozygous and one homozygous for the missense mutation G85E: a pancreatic sufficiency/insufficiency mutation with variable clinical presentation. J Med Genet 1996; 33: 823-828.

9. Chalkley G, Harris A. A cystic fibrosis patient who is homozygous for the G85E mutation has very mild disease. J Med Genet 1991; 28: 875-877.

10. De Braekeleer M, Daigneault J, Aubin G, et al. Phenotypic heterogeneity in $\mathrm{CF}$ sibs compound heterozygous for the G85E and 621+1G->T mutations. Clin Genet 1995; 47: 110111.

11. Kerem E, Nissim-Rafinat M, Augarten A, et al. A missense cystic fibrosis transmembrane conductance regulator mutation with variable phenotype. Pediatrics 1997; 100: E5.

12. European Thematic Network of Cystic Fibrosis. www. cfnetwork.be. Date last updated: December 21 2003. Date last accessed: February 162004.

13. Gibson LF, Cooke RE. A test for concentration of 
electrolytes in sweat in cystic fibrosis of the pancreas using pilocarpine by iontophoresis. Pediatrics 1959; 23: 545-549.

14. Prader A, Largo RH, Molinari L, Issler C. Physical growth of Swiss children from birth to 20 years of age. First Zurich longitudinal study of growth and development. Helv Pediatr Acta Suppl 1989; 52: 1-125.

15. Döring G, Conway SP, Heijerman HGM, et al. Antibiotic therapy against Pseudomonas aeruginosa in cystic fibrosis: a European consensus. Eur Respir J 2000; 16: 749-767.

16. American Thoracic Society. Standardization of spirometry 1987 update. Am Rev Respir Dis 1987; 136: 1285-1298.

17. Quanjer P, Porsboom GJJM. Spirometric reference values for white European children and adolescents: Polgar revisted. Pediatr Pulm 1995; 19: 135-142.

18. Wilschanski M, Zielenski J, Markiewicz D, et al. Correlation of sweat chloride concentration with classes of the cystic fibrosis transmembrane conductance regulator gene mutations. J Pediatr 1995; 127: 705-710.

19. Cuppens H, Lin W, Jaspers M, et al. Polyvariant mutant cystic fibrosis transmembrane conductance regulator genes: the polymorphic (TG)m locus explains the partial penetrance of the T5 polymorphism as a disease mutation. J Clin Invest 1998; 101: 487-496.

20. Kubesk P, Dörk T, Wulbrand U, et al. Genetic determinants of airways' colonisation with Pseudomonas aeruginosa in cystic fibrosis. Lancet 1993; 341: 189-194.

21. The Cystic Fibrosis Genotype-Phenotype Consortium: correlation between genotype and phenotype in patients with cystic fibrosis. N Engl J Med 1993; 329: 1308-1313.

22. Durno C, Corey M, Zielenski J, Tullis E, Tsui LC, Durie P. Genotype and phenotype correlations in patients with cystic fibrosis and pancreatitis. Gastroenterology 2002; 123: 1857-1864.
23. FitzSimmons SC. Cystic Fibrosis Foundation patient registry 1995: annual report. Bethesda, MD, Cystic Fibrosis Foundation, August 1996.

24. Durie P. Changes in the natural history of CF from a GI perspective. Plenary session at the Sixteenth Annual North American CF meeting, October 3-6, 2002. New Orleans. Ped Pulmonology: 2002, Suppl. 24, 35.

25. Kerem E, Corey M, Kerem B-S, et al. The relationship between genotype and phenotype in cystic fibrosis analysis of the most common mutation (DF508). N Engl J Med 1990; 323: $1517-1522$

26. Corey M, Durie P, Moore D, Forstner G, Levison H. Familial concordance of pancreatic function in cystic fibrosis. J Pediatr 1989; 115: 274-277.

27. Cutting G. Phenotype-genotype relationships. In: Cystic Fibrosis. 2nd Edn. Hodson ME, Geddes DM, eds. London, UK, Arnold Publishers, 2000; pp. 49-60.

28. Borgo G, Cabrini G, Mastella G, Ronchetti P, Devoto M, Romeo G. Phenotypic intrafamilial heterogeneity in cystic fibrosis. Clin Genet 1993; 44: 48-49.

29. Vankeerberghen A, Cuppens H, Cassiman JJ. The cystic fibrosis transmembrane conductance regulator: an intriguing protein with pleiotropic functions. J Cyst Fibrosis 2002; 1: 13-29.

30. Zielenski J, Corey M, Rozmahel R, et al. Detection of a cystic fibrosis modifier locus for meconium ileus on human chromosome 19q13. Nat Genet 1999; 22: 128-129.

31. Xiong X, Bragin A, Widdicombe J, Cohn J, Skach W. Structural cues involved in endoplasmic reticulum degradation of G85E and G91R mutant cystic fibrosis transmembrane conductance regulator. J Clin Invest 1997; 100: 1079-1088. 